\title{
Is The Theoretical Knowledge Reflected in Current Practice: Histopathological Helicobacter Pylori Research?
}

\author{
Mehmet Ali Kosekli* \\ Department of Gastroenterology, Abant Izzet Baysal University Hospital, Bolu, Turkey.
}

\begin{abstract}
Objective: Helicobacter pylori (H. pylori) is considered in the category of biological agent directly responsible for cancer. Guidelines recommend discontinuing suppressive conditions prior to histopathological helicobacter pylori testing, but there is little clinical trial data on how outcome is affected if this is not done. Optimal test recommendations in the guidelines are based on in vitro study results.

In present study, we aimed to observe whether there was a difference in the prevalence of $H$. pylori histopathologically in patients who met the necessary prerequisites before Esophago-Gastro-Duodenoscopy (EGD) compared to the subjects group who did not meet the PPI discontinuation condition.

Materials \& Methods: This retrospective study was conducted between October 2015 and August 2016 in a reference hospital with 1.5 million hinterlands. Patients who underwent EGD by meeting all the prerequisites recommended by the guidelines for the $H$. pylori test were included in the study group ( $\mathrm{n}=213$ ), and those who discontinued other suppressive drugs except PPI were included in the control group ( $\mathrm{n}=193$ ).
\end{abstract}

Results: While H. pylori was found to be $74.6 \%$ positive in the study group that provided all the prerequisites for histopathological $H$. pylori detection, $H$. pylori was found to be $64.9 \%$ positive in the control group continuing to receive PPI treatment $(\mathrm{p}=0.02)$.

Conclusion: In conclusion, we suggest that it is necessary to proceed with the guidance of classical knowledge in determining the presence of H. pylori, which is a common public health problem that is up-to-date.

Keywords: Helicobacter pylori, Suppressive condition, Proton pump inhibitor, Histopathology, Cancer, Guideline.

\section{INTRODUCTION}

Helicobacter pylori (H. pylori) ranks first among cancer related infections that in a study based on 2018 Globocan data [1]. When not eradicated, it is blamed in the aetiology of many diseases, from chronic gastritis to gastric maltoma, as a result of progressive damage to the mucosa, and is considered in the category of biological agent directly responsible for cancer by the World Health Organization [2-4].

The socioeconomic status, infrastructure and sanitation conditions of the countries affect its prevalence [5]. Non-invasive and invasive methods are used for $H$. pylori detection and the sensitivity and specificity of these methods vary [6-8]. Biopsy samples taken during esophagogastroduodenoscopy (EGD) show the presence / absence of $H$. pylori as well as the information about the gastric mucosa. In order to determine $H$. pylori histopathological, certain prerequisites must be met before EGD procedure. It is known that upper Gastrointestinal System (GIS) bleeding, antibiotics, bismuth preparations and Proton Pump Inhibitors (PPI) affect H. pylori tests. It is a common advice that PPIs should be discontinued for $H$. pylori detection, and it has been put into the guidelines after preclinical studies [9-11]. There are many studies on the negative effects of suppressive states on $H$. pylori tests. There are a

*Address correspondence to this author at the Department of Gastroenterology, Abant Izzet Baysal University Hospital, Bolu, Turkey.

Email:kosekli@gmail.com limited number of clinical studies directly investigating the effect of PPI use on histopathological H. pylori prevalence $[12,13]$.

In present study, it was investigated whether there was a difference in the prevalence of $H$. pylori histopathological in patients who met the necessary prerequisites before EGD compared to the subjects group who did not meet the PPI discontinuation condition and continued to use PPI.

\section{MATERIALS AND METHODS}

This retrospective study includes individuals who admitted to a reference hospital gastroenterology clinic between October 2015 and August 2016. Local ethic board approved the study. Consecutive patients enrolled to the study who admitted to the gastroenterology clinic without gastrointestinal bleeding, and with intact stomach. The patients were evaluated for study criteria right after obtaining at least 4 biopsy specimens from large and lesser curvatures of antrum and corpus. Suppressive conditions were noted by patient history and confirmed from computerized database (national patient data registry system). According to the guidelines, patients without suppressive conditions were enrolled to the study group while other subjects with only used PPI as a suppressive condition were grouped into the control group. Design of the study was abstracted in Fig. (1).

www.njhsciences.com 


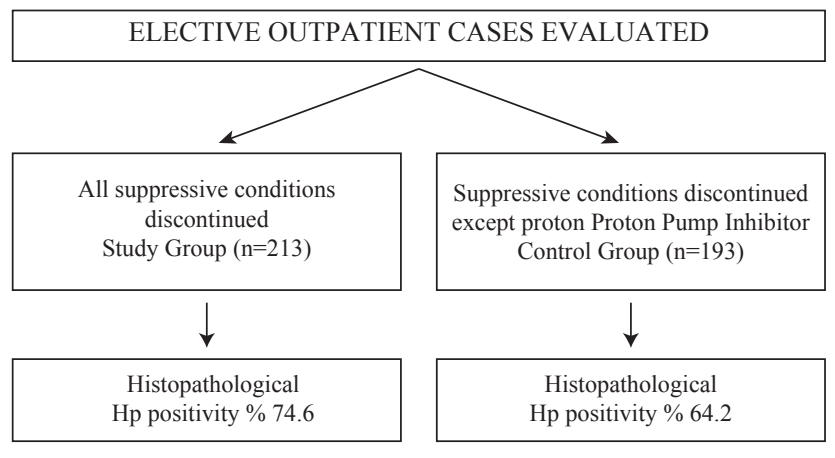

Fig. (1). Study Flowchart.

Exclusion criteria were as follows: unwilling to participate, inadequate data about medical history in last 1 month, conflicting medical history and national medical registry database.

Finally, 213 patients without a history of any suppressive conditions were enrolled in study group, while 193 patients with PPI use without antibiotic or bismuth usage were enrolled as controls. Endoscopic biopsy specimens were evaluated according to the Sydney classification after they have been transported in $10 \%$ formaldehyde solution to the pathology laboratory. Specimens were stained with Giemsa or hematoxylin eosin and $H$. pylori was evaluated with Toluidine dye [14].

\section{STATISTICAL ANALYSES}

SS 16.0 software was used in comparison of the data in study and control groups. Kolmogorov Smirnov test was used in evaluation of normality data. Data with normal distribution was compared with independent Samples T test and expressed as mean \pm standard deviation. Nonparametric data were expressed as numbers and percentage and compared with $\mathrm{X} 2$ test. A P value lower than 0.05 was considered as statistically significant.

\section{RESULTS}

Total population consisted of 406 patients; 213 in the study group (134 women (63\%), 79 men (37\%) and 193 subjects in the control group (106 women (55\%), 87 men (45\%)). Mean age of the study and control groups were $52 \pm 15$ years and $54.6 \pm 15$ years, respectively $(\mathrm{p}=0.360$ ). In the study group, 159 cases $(74.6 \%)$ were reported as positive for $H$. pylori, 54 cases $(25.4 \%)$ were reported to be $H$. pylori negative. The effect of gender on $H$. pylori has not been determined $(\mathrm{p}=0.100)$. Table 1 shows the characteristics of the study population.

H. pylori was positive in $124(64.2 \%)$ of 193 cases in the control group. There was no effect of gender on $H$. pylori positivity in patients in the control group $(\mathrm{p}=0.12)$.
Table 1. Effects of PPI use on H. pylori Positivity.

\begin{tabular}{|l|c|c|c|}
\hline Group & H. pylori $(+)$ & H. pylori $(-)$ & n \\
\hline PPI discontinued & $159(\% 74.6)^{*}$ & $54(\% 25.4)^{*}$ & 213 \\
\hline PPI continued & $124(\% 64.2)$ & $69(\% 35.8)$ & 193 \\
\hline $\begin{array}{l}\text { H. pylori: Helicobacter pylori, PPI: Proton pump inhibitor. } \\
* P=0.020 .\end{array}$ \\
\hline
\end{tabular}

While $H$. pylori was found to be $74.6 \%$ positive in the study group that provided all the prerequisites for histopathological H. pylori detection, $H$. pylori was found to be $64.2 \%$ positive in the control group continuing to receive PPI treatment. There is a statistically significant difference between the two groups in terms of $H$. pylori positivity $(\mathrm{p}=0.020)$.

\section{DISCUSSION}

The data underlying the guidelines for the detection of $H$. pylori are based on preclinical, in vitro studies. Our study is unique because it is a clinical study that aims to investigate the effect of PPI on histopathological $H$. pylori detection using real life data. While the prevalence of $H$. pylori was $74.6 \%$ in the study group performed under ideal conditions, the prevalence of H. pylori was found to be $64.2 \%$ lower in the control group whose PPI was not discontinued $(\mathrm{p}=0.02)$.

Contribution of $H$. pylori infection to gastric carcinogenesis; detection and treatment is important due to the importance of regression in $\mathrm{H}$. pylori -associated premalignant lesions with eradication [15]. Biopsy samples taken during esophagogastroduodenoscopy provide information about the presence of H. pylori as well as the histopathological findings of the mucosa. By histopathological method, H. pylori test has $90 \%$ sensitivity and $97 \%$ specificity, $96 \%$ positive and $97 \%$ negative predictive value $[16,17]$. Detection of $H$. pylori presence during the endoscopic biopsy procedure is affected by some factors. Therefore, it is recommended to stop antibiotic and bismuth preparations 1 month in advance and PPI group drugs 2 weeks before the procedure. In addition, the $H$. pylori test results of patients who had GIS bleeding in the last 2 weeks are affected by histopathological method [18]. It is difficult to provide optimal conditions in gastroenterology practice for various reasons.

The use of antibiotics and bismuth directly affects $H$. pylori with an antibacterial effect [19]. With the use of PPI, the colonization of $H$. pylori in the stomach changes, urease activity decreases and turns from spiral form to coccoid form [20, 21]. Therefore, the value of $H$. pylori tests decreases.

In a study conducted on $H$. pylori isolates, it was shown that omeprazole and lansoprazole exhibited a suppressive effect on $H$. pylori and urease activity, and these agents recovered the bacterial activity only 12-14 days after they were removed from the environment [22]. 
Knoop et al. found that $76 \%$ of the patients who underwent EGD in outpatients and inpatients were in suppressive condition and $70 \%$ of these cases were using PPI [13]. In the aforementioned study, $H$. pylori was investigated in half of the patients, and H. pylori was not indicated in the other half. In our study, due to the conditions of our country, a routine investigation of $H$. pylori was performed in all patients, and also in outpatient elective cases. Therefore, the cases are homogeneous in terms of $\mathrm{H}$. pylori research. In the study of Knoop et al. H. pylori positivity was $17.3 \%$ in the group that was deemed appropriate to investigate H. pylori. $82.7 \%$ of the cases were $H$. pylori negative and the rate of PPI usage in this negative group was investigated. In our study design, the effect of PPI use on $\mathrm{H}$. pylori is revealed more clearly since the cases are grouped in terms of PPI usage from the beginning.

In our study, while $H$. pylori negativity was $25.4 \%$ in the cases that met optimal conditions, it was found $35.8 \%$ negativity in the group using PPI. Therefore, the use of PPI has been shown to increase false negativity.

In the study conducted by Sirin et al. patients were recruited from 3 different centers and the areas where the biopsy were taken varied [12]. In our study, the patients were standardized by taking four biopsy samples from a single center and from 2 regions from all patients.

In a retrospective study conducted in Canada, the rate of standard biopsy from the antrum and corpus is $57 \%$ when the biopsied stomach segments of the cases undergoing $H$. pylori research are evaluated. The rate of taking biopsy only from the antrum is $47 \%$ and only from the corpus is $13 \%$ [23]. In this context, our study is a study in which both suppressive conditions and biopsy intake conditions are provided under optimal conditions. The lack of any method other than histopathology is the deficiency of our study. However, our clinical possibilities are limited with this method.

With this study, a result confirming the guideline information that PPI should be discontinued before EGD operation was reached. It has been shown that the false negativity rate of cases increases significantly when the procedure is performed without interrupting the PPI.

H. pylori infection is an issue that is up-to-date in gastroenterology practice, and eradication and vaccination efforts have not achieved the desired success. Despite the relative decrease in prevalence due to the interest of physicians and patients compared to the 90 's when it came to the agenda, it still remains a public health problem for many countries.

\section{CONCLUSION}

In conclusion, we suggest that it is necessary to proceed with the guidance of classical knowledge in determining the presence of $H$. pylori, which is a common public health problem that is up-to-date. It should be kept in mind that an invasive method with a sensitivity and specificity of more than $90 \%$ in detecting the presence of $H$. pylori results in a substantially false negative result when the necessary preconditions are not met.

\section{CONFLICT OF INTEREST}

Declared none.

\section{ACKNOWLEDGEMENTS}

Declared none.

\section{REFERENCES}

[1] de Martel C, Georges D, Bray F, Ferlay J, Clifford GM. Global burden of cancer attributable to infections in 2018: A worldwide incidence analysis. Lancet Global Health 2020; 8(2): e180-e90. DOI: 10.1016/S2214-109X(19)30488-7

[2] Correa P. Human gastric carcinogenesis: A multistep and multifactorial process--first American cancer society award lecture on cancer epidemiology and prevention. Cancer Res 1992; 52(24): 6735-40.

[3] Kikuchi S. Epidemiology of helicobacter pylori and gastric cancer. Gastric Cancer 2002; 5(1): 6-15. DOI: $10.1007 / \mathrm{s} 101200200001$

[4] Møller H, Heseltine E, Vainio H. Working group report on schistosomes, liver flukes and helicobacter pylori. Int J Cancer 1995; 60(5): 587-9. DOI: 10.1002/ijc.2910600502

[5] Peleteiro B, Bastos A, Ferro A, Lunet N. Prevalence of Helicobacter pylori infection worldwide: A systematic review of studies with national coverage. Dig Dis Sci 2014; 59(8): 1698-709. DOI: $10.1007 / \mathrm{s} 10620-014-3063-0$

[6] Versalovic J. Helicobacter pylori. Pathology and diagnostic strategies. Am J Clin Pathol 2003; 119(3): 403-12. DOI: 10.1309/5DTF5HT7NPLNA6J5

[7] Guarner J, Kalach N, Elitsur Y, Koletzko S. Helicobacter pylori diagnostic tests in children: Review of the literature from 1999 to 2009. Eur J Pediatr 2010; 169(1): 15-25. DOI: 10.1007/s00431-009-1033-x

[8] NIH Consensus Conference. Helicobacter pylori in peptic ulcer disease. NIH Consensus Development Panel on Helicobacter pylori in Peptic ulcer disease. JAMA 1994; 272(1): 65-9. DOI: 10.1001/jama.272.1.65

[9] Malfertheiner P, Megraud F, O'Morain CA, et al. Management of Helicobacter pylori infection-the Maastricht V/Florence Consensus Report. Gut 2017; 66(1): 6-30. DOI: 10.1136/gutjnl-2016-312288

[10] Sugano K, Tack J, Kuipers EJ, et al. Kyoto global consensus report on Helicobacter pylori gastritis. Gut 2015; 64(9): 
1353-67. DOI: 10.1136/gutjnl-2015-309252

[11] Chey WD, Wong BC. American College of Gastroenterology guideline on the management of Helicobacter pylori infection. Am J Gastroenterol 2007; 102(8): 1808-25. DOI: 10.1111/j.1572-0241.2007.01393.x

[12] Shirin D, Matalon S, Avidan B, Broide E, Shirin H Real-world Helicobacter pylori diagnosis in patients referred for esophagoduodenoscopy: The gap between guidelines and clinical practice. United European Gastroenterol J 2016; 4(6): 762-9. DOI: $10.1177 / 2050640615626052$

[13] Knoop RF, Petzold G, Amanzada A, et al. Testing of Helicobacter pylori by endoscopic biopsy: The clinical dilemma of suppressive conditions. Digestion 2020; 101(5): 552-6. DOI: $10.1159 / 000501270$

[14] Dixon MF, Genta RM, Yardley JH, Correa P. Classification and grading of gastritis: The updated Sydney system. Am J Surg Pathol 1996; 20(10): 1161-81. DOI: 10.1097/00000478-199610000-00001

[15] Ford AC, Forman D, Hunt RH, Yuan Y, Moayyedi P. Helicobacter pylori eradication therapy to prevent gastric cancer in healthy asymptomatic infected individuals: Systematic review and meta-analysis of randomised controlled trials. BMJ 2014; 348: g3174. DOI: 10.1136/bmj.g3174

[16] Amin Talebi Bezmin A. Diagnosis of Helicobacter pylori using invasive and noninvasive approaches. J Pathog 2018; 2018: 9064952. DOI: $10.1155 / 2018 / 9064952$

[17] Laheij RJ, de Boer WA, Jansen JB, van Lier HJ, Sneeberger
PM, Verbeek AL. Diagnostic performance of biopsy-based methods for determination of Helicobacter pylori infection without a reference standard. J Clin Epidemiol 2000; 53(7): 742-6. DOI: 10.1016/S0895-4356(99)00222-X

[18] Mégraud F, Lehours P. Helicobacter pylori detection and antimicrobial susceptibility testing. Clin Microbiol Rev 2007; 20(2): 280-322. DOI: 10.1128/CMR.00033-06

[19] McColl KE. Clinical practice. Helicobacter pylori infection. N Engl J Med 2010; 362(17): 1597-604. DOI: 10.1056/NEJMcp 1001110

[20] Berry V, Jennings K, Woodnutt G. Bactericidal and morphological effects of amoxicillin on Helicobacter pylori. Antimicrob Agents Chemother 1995; 39(8): 1859-61. DOI: 10.1128/AAC.39.8.1859

[21] She FF, Su DH, Lin JY, Zhou LY. Virulence and potential pathogenicity of coccoid Helicobacter pylori induced by antibiotics. World J Gastroenterol 2001; 7(2): 254-8. DOI: 10.3748/wjg.v7.i2.254

[22] Siavoshi F, Saniee P, Khalili-Samani S, et al. Evaluation of methods for $H$. pylori detection in PPI consumption using culture, rapid urease test and smear examination. Ann Transl Med 2015; 3(1): 11.

[23] El-Zimaity H, Serra S, Szentgyorgyi E, Vajpeyi R, Samani A. Gastric biopsies: The gap between evidence-based medicine and daily practice in the management of gastric Helicobacter pylori infection. Can J Gastroenterol 2013; 27(10): e25-30. DOI: $10.1155 / 2013 / 897423$ 